\title{
Amperean Pairing and the Pseudogap Phase of Cuprate Superconductors
}

\author{
Patrick A. Lee* \\ Department of Physics, Massachusetts Institute of Technology, Cambridge, Massachusetts 02139, USA
}

(Received 17 April 2014; published 29 July 2014)

\begin{abstract}
The enigmatic pseudogap phase in underdoped cuprate high- $T_{c}$ superconductors has long been recognized as a central puzzle of the $T_{c}$ problem. Recent data show that the pseudogap is likely a distinct phase, characterized by a medium range and quasistatic charge ordering. However, the origin of the ordering wave vector and the mechanism of the charge order is unknown. At the same time, earlier data show that precursive superconducting fluctuations are also associated with this phase. We propose that the pseudogap phase is a novel pairing state where electrons on the same side of the Fermi surface are paired, in strong contrast with conventional Bardeen-Cooper-Schrieffer theory which pairs electrons on opposite sides of the Fermi surface. In this state the Cooper pair carries a net momentum and belongs to a general class called pair density wave. The microscopic pairing mechanism comes from a gauge theory formulation of the resonating valence bond (RVB) picture, where spinons traveling in the same direction feel an attractive force in analogy with Ampere's effects in electromagnetism. We call this Amperean pairing. Charge order automatically appears as a subsidiary order parameter even when long-range pair order is destroyed by phase fluctuations. Our theory gives a prediction of the ordering wave vector which is in good agreement with experiment. Furthermore, the quasiparticle spectrum from our model explains many of the unusual features reported in photoemission experiments. The Fermi arc, the unusual way the tip of the arc terminates, and the relation of the spanning vector of the arc tips to the charge ordering wave vector also come out naturally. Finally, we propose an experiment that can directly test the notion of Amperean pairing.
\end{abstract}

DOI: 10.1103/PhysRevX.4.031017

Since the early days of cuprate superconductivity research, the pseudogap phase has been identified as a central piece of the high- $T_{c}$ puzzle [1]. The pseudogap opens below a temperature $T^{*}$ much above $T_{c}$ in underdoped cuprates, and it is visible in the spin susceptibility as detected by the Knight shift, in tunneling spectroscopy and in $c$-axis conductivity. Angle-resolved photoemission (ARPES) shows that the pseudogap opens in the antinodal region near $(0, \pi)$ (we set the lattice constant $a$ to unity), leaving behind ungapped "Fermi arcs" centered around the nodes. Recent x-ray scattering data [2-5] reveal that the pseudogap is likely to be a distinct phase, characterized by the onset of a charge density wave (CDW) with wave vectors at $(0, \pm \delta)$ and $( \pm \delta, 0)$, where $\delta$ decreases with increasing doping, thus confirming evidence for charge order found earlier by scanning tunneling microscope (STM) [6-9] and nuclear magnetic resonance (NMR) experiments [10]. Recent advances include STM and $\mathrm{x}$-ray studies on the same $\mathrm{Bi}_{2} \mathrm{Sr}_{2} \mathrm{CaCu}_{2} \mathrm{O}_{8+x}(\mathrm{Bi}-2212)$ samples [11]. Other signatures include Kerr rotation [12],

\footnotetext{
*palee@mit.edu
}

Published by the American Physical Society under the terms of the Creative Commons Attribution 3.0 License. Further distribution of this work must maintain attribution to the author(s) and the published article's title, journal citation, and DOI.
Subject Areas: Condensed Matter Physics,

Superconductivity

the emergence of anisotropy in the Nernst effect, etc. [13]. (Another set of experiments found the onset of intra-unit cell magnetization (loop currents) at a somewhat higher temperature [14]. We shall not address this phenomenon in this paper.)

The CDW is enhanced by a magnetic field and appears to be connected to the high-magnetic-field state where quantum oscillations have been observed [15]. Indeed, recent work [16] claims that the intrinsic $H_{c 2}$ of some underdoped $\mathrm{YBa}_{2} \mathrm{Cu}_{3} \mathrm{O}_{6+x}$ (YBCO) samples may be as low as $22 \mathrm{~T}$, a shockingly low energy scale compared with the energy gap scale as well as with $T_{c}$. At the same time, the onset of superconductivity causes a reduction of the CDW amplitude [2-4], suggesting that the pseudogap should be considered a competing phase. However, up to now, the identity of this phase is not at all clear because the data seem to give conflicting signatures. Certain features of the pseudogap state suggest the presence of short-range superconducting order. For example, diamagnetic fluctuations are observed much above $T_{c}$ [17], and the spectral weight of the Drude conductivity between members of the bilayers in YBCO is found to increase below about $180 \mathrm{~K}$, a trend consistent with fluctuating superconductivity and opposite to gap formation due to charge order [18]. On the other hand, the CDW is rather long ranged in the plane, but it does not resemble a conventional CDW driven by Fermi 
surface nesting. A recent comparison of $\delta$ with the nesting vector connecting the Fermi surfaces at the antinodal points in single-layer $\mathrm{Bi}_{2} \mathrm{Sr}_{2-x} \mathrm{La}_{x} \mathrm{CuO}_{6+\delta}(\mathrm{Bi} 2201)$ found that $\delta$ is larger by about a factor of 2 . Instead, the CDW vector was suggested to connect the ends of the Fermi arcs where the gap is zero or very small [5]. This directly contradicts the standard CDW picture where an energy gap is expected at precisely that point (see Appendix A). As we discuss in greater detail later, this difficulty was anticipated in an earlier study, where detailed ARPES data show that the spectrum contains highly anomalous features that cannot be understood with a CDW model [12]. In this paper, we propose a new model for the pseudogap state: In this picture, the pseudogap state is indeed a competing phase, but unlike previous proposals, it is a novel kind of superconductor that is destroyed by phase fluctuations and CDW is a subsidiary order. In this way, the apparently conflicting data described above fall naturally into a consistent framework.

Our model is based on the idea of Amperean pairing introduced earlier in the context of quantum spin liquids [19]. The U(1) spin liquid is described by a spinon Fermi surface coupled to a $\mathrm{U}(1)$ gauge field. In this system, the coupling to a transverse gauge field (gauge magnetic field) is strong and unscreened. Just as in electrodynamics, the current of a carrier produces a gauge magnetic field that creates an attractive force to another carrier moving in the same direction, due to the Ampere effect. The transverse gauge-field-mediated interaction contributes the following to the action [19]:

$$
\begin{aligned}
S_{\mathrm{int}}= & -\frac{1}{2 v \beta} \sum_{\boldsymbol{p}_{1} \boldsymbol{p}_{2}, \boldsymbol{q}} D(q)\left(\boldsymbol{v}_{\boldsymbol{p}_{1}} \times \hat{q}\right) \\
& \cdot\left(\boldsymbol{v}_{\boldsymbol{p}_{2}} \times \hat{q}\right) f_{\boldsymbol{p}_{1}+\boldsymbol{q}, \sigma}^{\dagger} f_{\boldsymbol{p}_{2}-\boldsymbol{q}, \sigma^{\prime}}^{\dagger} f_{\boldsymbol{p}_{2}, \sigma^{\prime}} f_{\boldsymbol{p}_{1}, \sigma},
\end{aligned}
$$

where $v$ is the volume, $\beta=1 / \mathrm{k} T, v_{p}$ is the velocity of the spinon with momentum $\boldsymbol{p}, D(\boldsymbol{q})$ is the gauge-field propagator, and $f_{\boldsymbol{p}, \sigma}$ is the spinon destruction operator with spin $\sigma$. In Ref. [19], we showed that because of the singular nature of the gauge propagator, the Fermi surface is unstable to a special kind of pairing where particles in the vicinity of a given spot $\boldsymbol{K}$ on the Fermi surface form Cooper pairs. This is radically different from conventional BCS pairing, which pairs particles on opposite sides of the Fermi surface. We call this Amperean pairing.

In one version of the RVB theory of high- $T_{c}$ superconductors, a spinon Fermi surface is formed that is coupled to a $U(1)$ gauge field [1]. Unlike the spin liquid, there is also a gapless charge degree of freedom that is described by a bosonic holon coupled to the same gauge field. The bosons tend to condense and convert the spinons to electrons with a reduced spectral weight equal to $p$, the hole-doping concentration. While the gauge field is gapped by the Anderson-Higgs mechanism, the gap is small for small $p$ and gauge fluctuations remain important over a large temperature range, which is referred to as the incoherent Fermi liquid region [20]. Since it is in this region that the pseudogap state is formed, it is reasonable to assume that the same Amperean mechanism is at work and Eq. (1) still applies. Up to now, the standard RVB picture is that $d$-wave pairing of spinons sets in below a certain temperature [21]. Instead, we assume that the Amperean pairing has a slightly lower free energy and preempts the $d$-wave pairing. This is reasonable because many states are competitive in energy with the $d$-wave state, including pairdensity-wave states which share common properties with Amperean pairing as discussed below [22]. As we shall see, Amperean pairing leaves segments of gapless excitations that contribute to a $T$ linear entropy, exceeding the $T^{2}$ term for $d$-wave pairing. Consequently, even if $d$-wave pairing is the true ground state, the Amperean state can have a lower free energy above some temperature.

In the cuprate, it is natural to view the hole Fermi surface as the analog of the large and almost circular Fermi surface in the spin-liquid problem. As shown in Fig. 1, we choose the points $\boldsymbol{K}$ and $-\boldsymbol{K}$ [it is convenient to measure these momenta from $(0, \pi)$ ] on the Fermi surface at the antinode as the points of Amperean pairing. Let us introduce the mean-field decoupling of Eq. (1),

$$
\begin{aligned}
S^{\mathrm{MF}}= & \Delta_{2 \boldsymbol{K}}^{*}(\boldsymbol{k}) f_{\boldsymbol{k} \uparrow} f_{-\boldsymbol{k}+2 \boldsymbol{K} \downarrow}+\text { c.c. } \\
& +\Delta_{-2 \boldsymbol{K}}^{*}(\boldsymbol{k}) f_{\boldsymbol{k} \uparrow} f_{-\boldsymbol{k}-2 \boldsymbol{K} \downarrow}+\text { c.c., }
\end{aligned}
$$

where we have set $\sigma=\downarrow, \sigma^{\prime}=\uparrow, \boldsymbol{p}_{2}=\boldsymbol{k}, \boldsymbol{p}_{1}=-\boldsymbol{k} \pm 2 \boldsymbol{K}$ in Eq. (1) and $\Delta_{ \pm 2 \boldsymbol{K}}(\boldsymbol{k})$ comes from the remainder of the equation and is proportional to a sum over $\boldsymbol{k}^{\prime}$ of $\left\langle f_{\boldsymbol{k}^{\prime} \uparrow} f_{-\boldsymbol{k}^{\prime} \pm 2 \boldsymbol{K} \downarrow}\right\rangle$. All momenta are defined up to a reciprocal lattice vector. Instead of attempting a self-consistent solution of the mean-field equation, in this paper we simply assume a reasonable form of $\Delta_{ \pm 2 \boldsymbol{K}}(\boldsymbol{k})$ and explore the resulting quasiparticle structure. This is an essential first

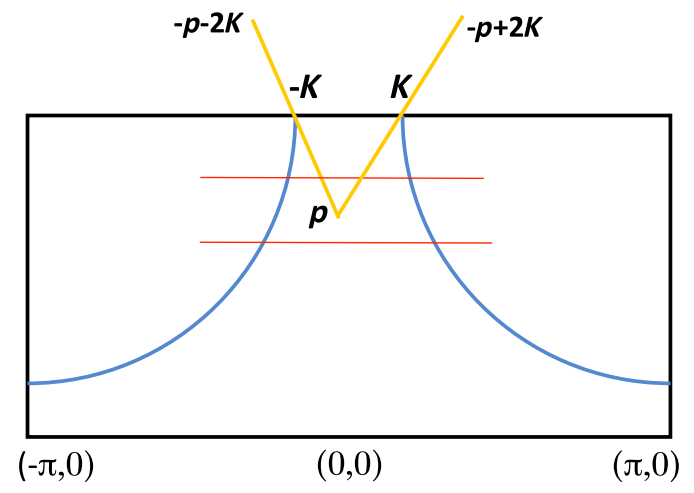

FIG. 1. Schematic representation of the top half of the cuprate Brillouin zone and the Fermi surface. Pairs are formed between fermions near $\boldsymbol{K}$ with momenta $\boldsymbol{p}$ and $-\boldsymbol{p}+2 \boldsymbol{K}$ and similarly near $-\boldsymbol{K}$. The Cooper pairs carry momenta $\boldsymbol{Q}=2 \boldsymbol{K}$ and $-2 \boldsymbol{K}$, respectively. Red lines mark some of the scans shown in Fig. 3. 
step because the quasiparticle spectrum is quite different from our intuition based on the conventional BCS pairing. Our ansatz is

$$
\Delta_{ \pm 2 \boldsymbol{K}}(\boldsymbol{k})=f\left(k_{y}\right) \Delta_{0},
$$

where $f\left(k_{y}\right)=e^{-\left(\pi-k_{y}\right)^{2} / k_{0}^{2}}$, with $k_{0}$ chosen to represent the fact that the pairing should be limited to the vicinity of $\boldsymbol{K}$.

We see from Eq. (2) that the pairing order parameters carry a total momentum of $2 \boldsymbol{K}$ or $-2 \boldsymbol{K}$ [23]. This is because the pair is made up of two fermions moving in the same direction and the total momentum is $\pm 2 \boldsymbol{K}$. By choosing $\Delta_{2 K}$ and $\Delta_{-2 K}$ to have the same amplitude, the order parameter is modulated in space as $\Delta(\boldsymbol{r}) \approx \cos (2 \boldsymbol{K} \cdot \boldsymbol{r})$. This belongs to a general class of pairing, which has been named pair density wave (PDW) [24,25]. Historically, the first example of PDW is the Fulde-Ferrell-Larkin-Ovchinnikov state (or, more precisely, the LO state), where the PDW is argued to be more stable than the uniform state when the Fermi surfaces are split by Zeeman splitting [26,27]. More recently, another example has been introduced in connection with stripe formation in the $\mathrm{La}_{2-x} \mathrm{Ba}_{x} \mathrm{CuO}_{4}$ (LBCO) system near doping $p=1 / 8$ [25]. We shall refer to this as stripe PDW. In this case, the spins form a spin-density wave with wave vector $Q=2 \pi / 8 a$, which is interpreted as antiphase Neel states separated by charged domain walls which produces charge order at wave vector $2 Q=2 \pi / 4 a$. The superconductor is assumed to be modulated at the same $Q$ and interpreted as $d$-wave superconductors with antiphase domains. The state was introduced to explain the observation that while a superconducting state exists much above $T_{c}$ within each layer, the layers fail to order coherently $[28,29]$. Indeed, the stripe PDW has been shown

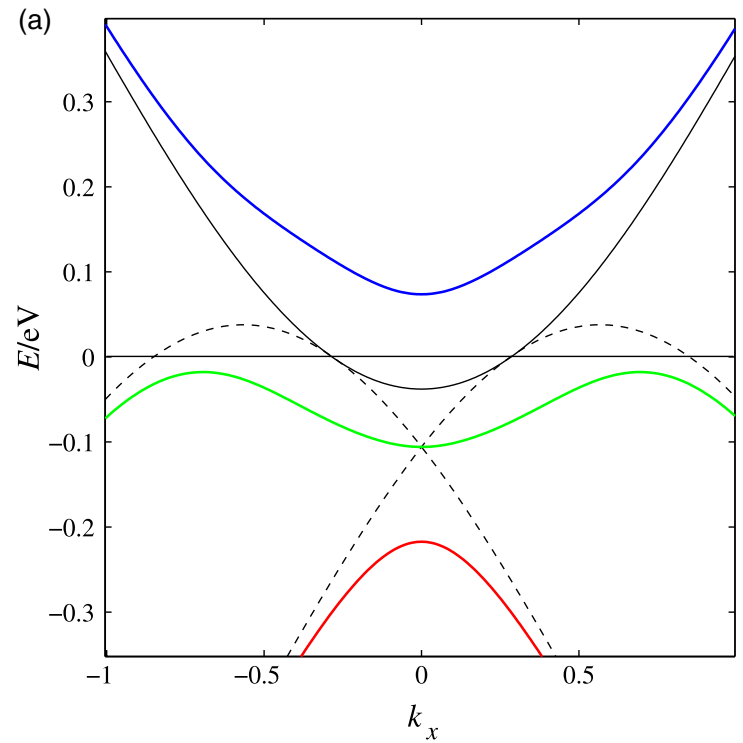

to be energetically competitive in earlier projected wavefunction studies [30] and has been suggested as being stabilized in high magnetic fields [31,32]. We note that the microscopic picture of this state is very different from our Amperean pairing state. The stripe PDW begins with the stripe picture of the SDW and, up to now, has been discussed only in connection with the stripe phenomena near $p=1 / 8$. The Amperean pairing picture is not associated with any SDW order. Instead, the wave vector is given in terms of Fermi surface spanning vectors, which decrease with increasing doping, a trend opposite to that of stripe PDW. The main driving force is the pairing energy, which can be a high-temperature scale. We may refer to our state as $2 k_{F}$ PDW. On the other hand, like the stripe PDW, there is a CDW with a period $2 Q=4 K$ associated with Amperean pairing. It is quite possible that Amperean pairing helps stabilize the stripe-PDW state in certain materials, such as LBCO, which favor SDW ordering and stripe formation. The stripe PDW is then separated from the high-temperature Amperean pair state by the phase transition near $50 \mathrm{~K}$. We emphasize that in our view, Amperean pairing is the driver and the $\mathrm{CDW}$ is a subsidiary order parameter.

Let us first consider $\boldsymbol{k}$ in the vicinity of $\boldsymbol{K}$ and calculate the spectrum due to the coupling of $c_{k \uparrow}$ and $c_{-k+2 \boldsymbol{K} \downarrow}^{\dagger}$ in the presence of $\Delta_{2 K}$. Diagonalization of a $2 \times 2$ matrix gives

$$
E_{k \uparrow}^{ \pm}=\frac{1}{2}\left(\xi_{k}-\xi_{-k+\boldsymbol{Q}}\right) \pm \sqrt{\frac{1}{4}\left(\xi_{k}+\xi_{-k+\boldsymbol{Q}}\right)^{2}+\left|\Delta_{\boldsymbol{Q}}\right|^{2}}
$$

where $\xi_{k}=\varepsilon_{k}-\mu$ and $\boldsymbol{Q}=2 \boldsymbol{K}$. Equation (4) replaces the familiar BCS spectrum $\pm \sqrt{\xi_{\boldsymbol{k}}^{2}+\left|\Delta_{\boldsymbol{Q}}\right|^{2}}$ and is no longer

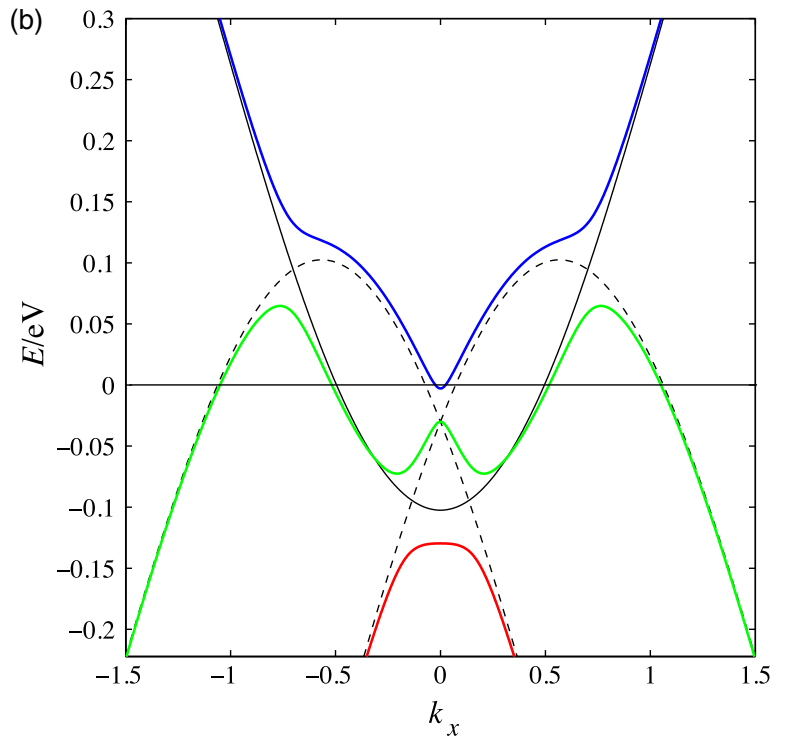

FIG. 2. Plot of the energy bands and the bare dispersion $\varepsilon_{k}-\mu$ versus $k_{x}$ for (a) $k_{y}=\pi$ and (b) $k_{y}=\pi-1.0$, using parameters appropriate for Bi-2201 [12]. The dashed lines show $-\left(\varepsilon_{\mathbf{k} \pm 2 \mathbf{K}}-\mu\right)$. Turning on $\Delta_{0}=100 \mathrm{meV}$ with $k_{0}=1.0 \mathrm{in} \mathrm{Eq.} \mathrm{(3)} \mathrm{splits} \mathrm{the} \mathrm{bands}$ and creates an energy gap for $k_{y}=\pi$. The gap vanishes for $k_{y}=\pi-1.0$, creating a Fermi arc. 
(a)

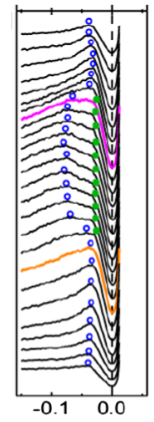

(f) $\delta k_{y}=0$

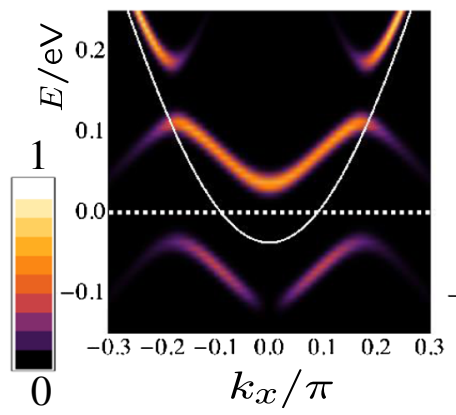

$\delta k_{y}=0$

(b)

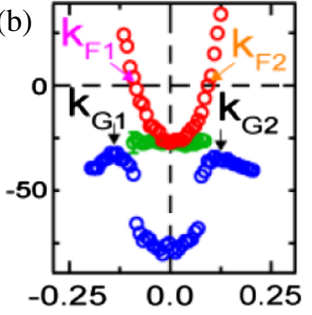

$\delta k_{y}=0.6$

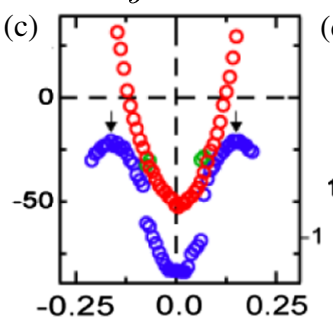

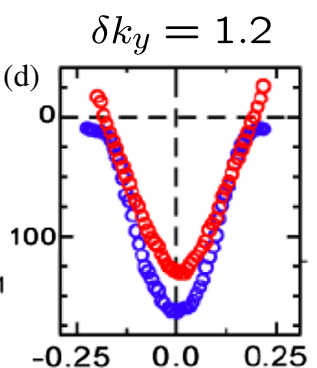

(h) $\delta k_{y}=0.9$

(g) $\delta k_{y}=0.6$

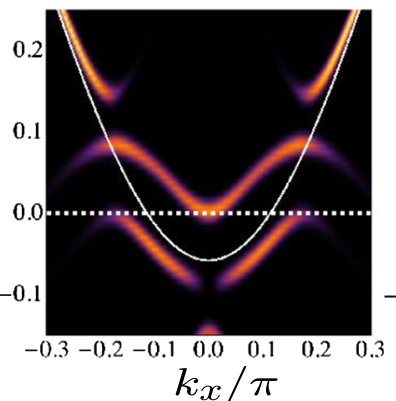

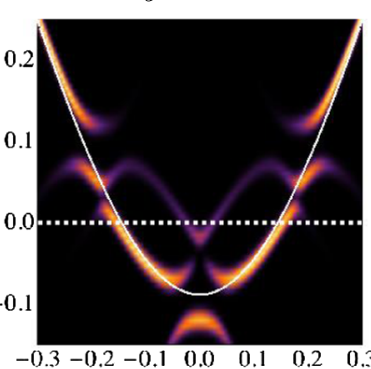

$k_{x} / \pi$

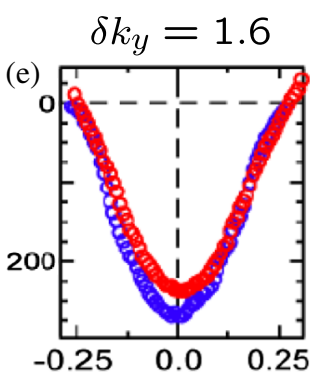

(i) $\delta k_{y}=1.2$

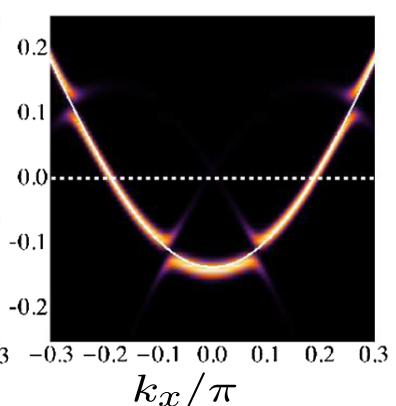

FIG. 3. ARPES spectra from Ref. [12] for scans with approximately constant $k_{y}=\pi-\delta k_{y}$, where $\delta k_{y} \approx 0,0.6,1.2$, and 1.6 . Raw data for $\delta k_{y}=0$ are shown in (a). The Fermi arc begins near $\delta k_{y}=1.2$. The Amperean pairing spectra are shown $(f-i)$ for $\delta k_{y}=0,0.6,0.9$, and 1.2 using $\Delta_{0}=75 \mathrm{meV}$ and $k_{0}=1$. The white line shows the band before pairing. With these parameters, the gap closes and the Fermi arc begins near $\delta k_{y}=0.6$. The negative energy states are to be compared with the data. In (f), we note that the minimum excitation energy to the Fermi level does not lie at $k_{F}$ but at a larger wave vector. This feature has been noted by the experimentalists, who introduced the wave vector $k_{G}$ in their figures (b) and (c) to describe this. The green dots in (b) appear only below $T_{c}$, and the blue dots at the band bottom mark the peak of a very broad spectrum near $100 \mathrm{meV}$ as seen in (a). The red dots in (b)-(e) are data taken above the pseudogap temperature.

particle-hole symmetric. In Fig. 2, we include, in addition, the coupling to $c_{-\boldsymbol{k}-2 \boldsymbol{K} \downarrow}^{\dagger}$ to restore inversion symmetry. We plot the band dispersions as well as the bare bands $\xi_{k}$ and $-\xi_{-\boldsymbol{k} \pm \boldsymbol{Q}}$ as a function of $k_{x}$ for $k_{y}=\pi$ [33]. Note that the bare bands touch each other at the Fermi level with parallel tangents. Indeed, if we ignore the band curvature and approximate $\xi_{k}=v_{F}\left(k-k_{F}\right)$ in Eq. (2), the two bands are exactly degenerate and are split by $\left|\Delta_{Q}\right|$ for all $k_{x}$. This gap opening is the source of energy gain of the Amperean pairing state. Note that in this approximation, the Fermi surface remains gapless: The level splitting simply splits the Fermi vector [34]. However, once the band curvature is included and $\left|\Delta_{Q}\right|$ is large enough, a gap is opened at the Fermi level near $(0, \pi)$, as shown in Fig. 2(a). As $k_{y}$ moves away from $\pi / a$, the degeneracy is lifted and the effect of $\Delta_{Q}$ diminishes, and eventually the Fermi surface is restored, as seen in Fig. 2(b). This mechanism produces the Fermi arc. (Strictly speaking, the quasiparticles at the Fermi energy form a closed pocket, but it resembles an arc because the spectral weight on the backside of the pocket is very small.) Importantly, the gap closes by occupied states arising up from below the Fermi energy, in contrast with a gap produced by CDW (see Appendix A). We next consider the coupling between five states, $\left(c_{k \uparrow}, c_{-k+\boldsymbol{Q} \downarrow}^{\dagger}, c_{-k-\boldsymbol{Q} \downarrow}^{\dagger}, c_{\boldsymbol{k}+2 \boldsymbol{Q} \uparrow}, c_{k-2 \boldsymbol{Q} \downarrow}\right), \quad$ and diagonalize the following $5 \times 5$ matrix:

$$
\left[\begin{array}{ccccc}
\xi_{k} & \Delta_{Q} & \Delta_{-Q} & C_{2 Q} & C_{-2 Q} \\
\Delta_{Q}^{*} & -\xi_{-k+Q} & 0 & 0 & \Delta_{-Q}^{*} \\
\Delta_{-Q}^{*} & 0 & -\xi_{-k-Q} & \Delta_{Q}^{*} & 0 \\
C_{2 Q}^{*} & 0 & \Delta_{Q} & \xi_{k+2 Q} & 0 \\
C_{-2 Q}^{*} & \Delta_{-Q} & 0 & 0 & \xi_{k-2 Q}
\end{array}\right] .
$$

We added $c_{\boldsymbol{k} \pm 2 \boldsymbol{Q}, \uparrow}$, which are coupled to $c_{\boldsymbol{k} \uparrow}$ with the matrix element $C_{ \pm 2 Q}$ due to the CDW order, but more importantly, they are also coupled to $c_{-k \pm \boldsymbol{Q}, \downarrow}^{\dagger}$. As seen from the full spectra in Appendix B, the latter bands are also degenerate at the Fermi level and give rise to splitting. Inclusion of this coupling helps create a pseudogap in the tunneling density of states which is closer to being particle-hole symmetric, as shown later.

In Figs. 3(f)-3(i), we show a series of band dispersion with color intensity proportional to the spectral weight $\left|\nu_{\alpha}\right|^{2}$, where $\alpha=1, \ldots, 5$ labels the bands and $\nu_{\alpha}$ is the amplitude of the state $c_{k \uparrow}$ for eigenstate $\alpha$. The states below the Fermi level in this plot can be directly compared with 
ARPES data, which are reproduced in Fig. 3 for Bi2201. As discussed in Ref. [12], the spectra show two highly unusual features. While a gap is seen at the Fermi level for $k_{y}$ near $\pi$, the gap maximum is not at the Fermi momentum $k_{F}$ determined at high temperatures as expected for BCS pairing. Instead, the band extends beyond $k_{F}$ and shows a peak at $k_{G}$ before losing spectral weight. As seen in Figs. 3(f)-3(h), this unusual feature is captured by the Amperean pairing model. As $k_{y}$ increases, this effectively pushes the Fermi level crossing somewhat beyond the $k_{F}$ of the original band. As shown in Fig. 4, this gives rise to a bending of the Fermi surface contour near the tip of the arc away from the high-temperature Fermi surface, which is often seen in ARPES as well as STM data [35]. A more detailed examination of the spectrum in Appendix B shows that near the antinodes, the top of the occupied band lies near $k_{x}= \pm Q$. If we define the tip of the Fermi arc as the $\boldsymbol{k}$ point where the top of the occupied band meets the Fermi level, this happens at $k_{x}= \pm Q$. Thus, the vector connecting the tips of the arcs is the CDW wave vector, an observation made empirically by Comin et al. [5]. Due to the fact that the spectral weight is stronger for $\left|k_{x}\right|<Q$, the tip is rather ill defined in Fig. 4 and tends to lie at $\left|k_{x}\right|$ slightly less than Q. Finally, as seen in Fig. 3(g), near the tip of the Fermi arc the band dispersion is not particle-hole symmetric but simply turns around and loses spectral weight. This feature has been emphasized by Yang et al. [36] in their data, which they interpreted using a phenomenological model by Yang et al. [37].

A second unusual feature of the data of Ref. [12] is that near $k_{y}=\pi$, a broad spectral weight emerges around

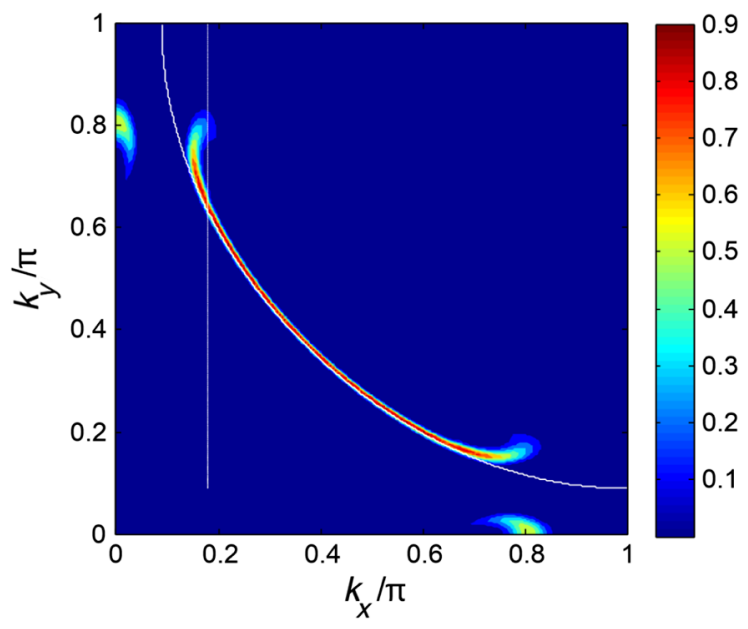

FIG. 4. The spectral weight of the bands (Gaussian broadened with a $10-\mathrm{meV}$ width) at the Fermi level. Parameters are the same as in Fig. 3, which correspond to a doping density $p=0.207$. The white line shows the bare Fermi surface. The vertical line marks $k_{x}=\mathrm{Q}$. Note that the tip of the Fermi arc is close to this line; hence, the wave vector connecting the Fermi arcs has length near $2 \mathrm{Q}$, which is the CDW wave vector in our theory. This figure has been symmetrized about the diagonal to account for PDW order in both the $\mathrm{x}$ and $\mathrm{y}$ directions.
$-100 \mathrm{meV}$, far below the band bottom at $-35 \mathrm{meV}$. This feature persists to large $\delta k_{y}$ and gives rise to the unusual feature that the spectrum appears broader at low temperatures than above $T^{*}$. In a conventional CDW model (see Appendix A), it is very hard to see where this spectral weight comes from. In the Amperean pairing model, the band is connected to the crossing of the $-\boldsymbol{k} \pm \boldsymbol{Q}$ hole bands located near $100 \mathrm{meV}$. We expect these highly excited quasiparticles to be strongly coupled, and the scattering between them can give rise to a broad line shape, as seen in experiment. Physically, these states should be interpreted as Andreev-reflected hole states with momentum shifted by $\pm \boldsymbol{Q}$ because of the Amperean pairing amplitude $\Delta_{ \pm} \boldsymbol{Q}$.

We see from Fig. 3(f) that the states at the saddle point $\left(k_{x}=0\right)$ are pushed above the Fermi level. In Fig. 3(g) this band just touches the Fermi level. When we couple to a conventional uniform BCS order parameter, these states will be split and produce coherent peaks in the usual way. This is consistent with the feature in the data marked by the green dots. These states are the origin of the weight seen near $\left(k_{x}, k_{y}\right) / \pi=(0,0.8)$ and $(0.8,0)$ in Fig. 4 . While these states at the Fermi level have not been seen experimentally as sharp features, they may be broadened by lifetime effects and just contribute to a broad background above $T_{c}$. It is also not clear how much the existence of these weights depends on the details of the ansatz. The more robust consequence of our model is the existence of the unoccupied band seen in Fig. 3(f) and 3(g). It may be possible to search for these unoccupied bands by STM quasiparticle interference measurements. The transition between the occupied and unocccupied bands may also contribute to features in optical absorption.

In Fig. 3, we have set the CDW coupling $C_{2 Q}=0$. It turns out that including a finite $C_{2 Q}$ does not significantly increase the energy gap and has little effect on the spectrum near the Fermi energy except that it opens a gap at the crossing of the vertical white line and the bare Fermi surface shown in Fig. 4 . This is because $C_{2}$ couples the original bands far above the Fermi energy. The observed pseudogap is almost entirely due to Amperean pairing. As discussed in Appendix A, it is not possible to explain the ARPES data in models where CDW alone is the driving force behind the energy gap.

In Fig. 5, we show the tunneling density of states

$$
\nu(E)=\sum_{k, \alpha}\left|\nu_{\alpha}\right|^{2} \delta\left(E-E_{\alpha}\right)
$$

It shows a pseudogap with some asymmetry. After lifetime broadening, it resembles STM tunneling data.

The density operator $\rho_{2 \boldsymbol{Q}}=\sum_{\sigma} c_{\boldsymbol{k}-2 \boldsymbol{Q}, \sigma}^{\dagger} c_{\boldsymbol{k}, \sigma}$ has a nonzero average in mean-field theory; i.e., $\left\langle\rho_{2 Q}\right\rangle$ is an induced order parameter. Its physical interpretation in the case of spin liquid is a modulation of the singlet valence bond strength, i.e., an incommensurate valence bond solid [19]. For the 


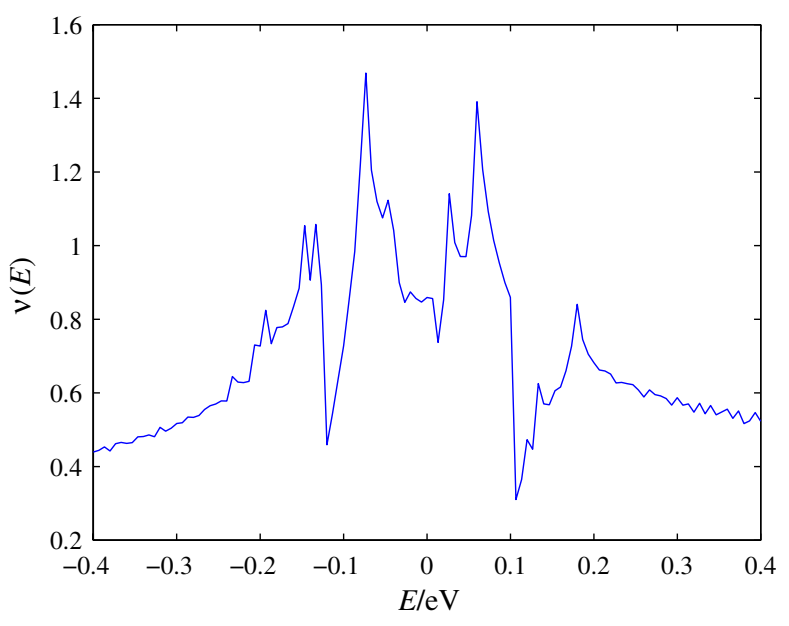

FIG. 5. Plot of the tunneling density of state $\nu(E)$ for the same parameters as in Fig. 3.

superconductor, this gives rise to a CDW. Our theory predicts the CDW vector to be twice the PDW vector, which we set to be the vector spanning the Fermi surface at the antinodes. We have focused on $(0, \pi)$, where the spanning vector is $\boldsymbol{Q}_{1}=(Q, 0)$. Similar consideration near $(\pi, 0)$ gives $\boldsymbol{Q}_{2}=(0, Q)$. Since the two antinodal regions are far apart and relatively independent, we expect the pairing at the two nodes to be weakly coupled and to coexist. Thus, our picture favors the bidirectional CDW (checkerboard) rather than unidirectional stripes, in agreement with the pattern seen by STM on Bi-2201 [9]. In Fig. 6, we compare the predicted CDW ordering vector $2 Q$ with a collection of measurements on Bi2201, and the agreement is satisfactory. Note that in our theory, $2 \mathrm{Q}$ is also correlated with the wave vector connecting the tip of the arcs, in agreement with the empirical observation by Comin et al. [5]. It will be good to make a similar comparison

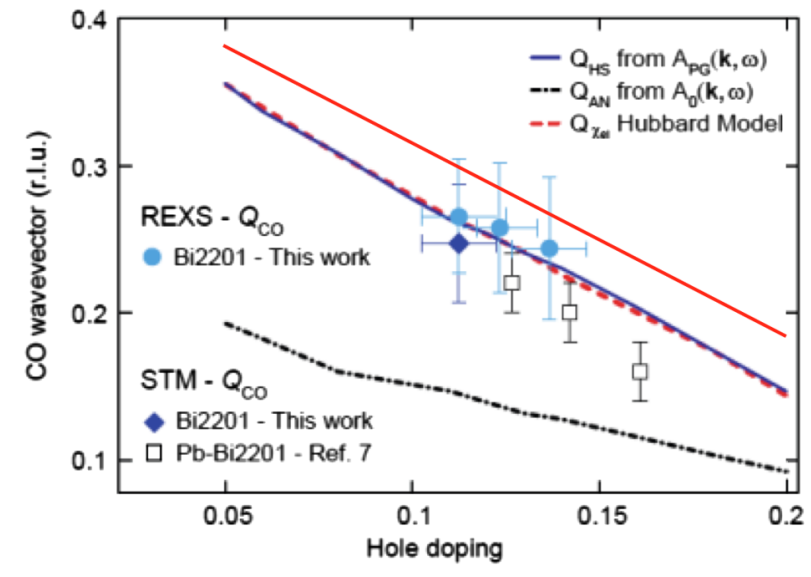

FIG. 6. A collection of data for the CDW wave vector $\delta$ (in units of $2 \pi$ ) vs hole-doping concentration on the Bi2201 system, taken from Ref. [5]. The dashed dotted line marked $Q_{\mathrm{AN}}$ is the Fermi surface spanning vector at the antinode, which corresponds to $Q$ in our notation. The solid red line is approximately $2 Q_{\mathrm{AN}}$, which is the prediction of the Amperean pairing model. using the data on Bi2212 [11], if accurate data on the antinodal spanning vectors are available.

In materials with intrinsic anisotropy due to chains such as YBCO, it is found that the CDW scattering integrated intensity shows an anisotropy $[38,39]$ which is presumably responsible for the nematic behavior in transport measurements such as the Nernst effect [13]. We believe that CDW with both $\boldsymbol{Q}_{1}$ and $\boldsymbol{Q}_{2}$ coexist in a given region but with different strengths. As discussed below, this coexistence is needed to understand the quantum oscillation data.

By leaving segments of the Fermi surface ungapped, the Amperean paired state has a finite normal fluid density even at zero temperature and hence a smaller superfluid density $\rho_{s}$ compared with the uniform state. We assume phase fluctuations and the nucleation of vortex-antivortex pairs suppress phase coherence, so that pairing is only short-range ordered until the conventional $d$-wave order takes over and gaps out the Fermi arc. The competition for the Fermi surface leads to the reduction of Amperean pairing below $T_{c}$, as clearly seen in x-ray experiments. A schematic phase diagram is shown in Fig. 7, where a shortrange-ordered PDW state is the dominant feature. As a competing phase, it is natural for this state to form a large vortex core when the $d$-wave superconductor is subject to a magnetic field. This explains the observation of checkerboard patterns near the vortex core by STM some time ago [40]. At a relatively low field, these cores overlap. As shown in Fig. 7(b), the small critical field [16] marks the transition to the short-range-ordered Amperean pairing state. The phase diagram also shows a long-range-ordered CDW state forming out of the short-range-ordered PDW, as explained in the next section. In real materials, the CDW is short-range ordered because of disorder pinning. We follow Harrison and Sebastian [41] and use the $2 \boldsymbol{Q}_{1}$ and $2 \boldsymbol{Q}_{2}$ vectors to connect pieces of the Fermi arcs, giving rise to a small pocket which may be the origin of the quantum oscillations with electronlike carriers. The recent observation of quantum oscillation and $\mathrm{CDW}$ in the $\mathrm{Hg} 1201$ compound found a pocket area that is larger than that of YBCO, while the CDW wave vector is shorter [42]. The trend supports this scenario.

Next, we discuss the appearance of Kerr rotation below $T^{*}$. A recent paper raised the possibility that the Kerr effect is due to a gyrotropic effect that requires the breaking of reflection symmetry in all directions, as opposed to the breaking of time-reversal symmetry as originally assumed [43]. While this claim has been withdrawn [44], we show here, for completeness, that our scenario can lead to a gyrotropic state.

Let us define the phases of the various order parameters as $\Delta_{ \pm \boldsymbol{Q}_{\alpha}}=\left|\Delta_{ \pm \boldsymbol{Q}_{\alpha}}\right| \exp \left(i \theta_{ \pm \boldsymbol{Q}_{\alpha}}\right), \rho_{2 \boldsymbol{Q}_{\alpha}}=\left|\rho_{2 \boldsymbol{Q}_{\alpha}}\right| \exp \left(i \phi_{\alpha}\right), \alpha=$ 1,2 . If $\left|\Delta_{\boldsymbol{Q}_{\alpha}}\right|=\left|\Delta_{-} \boldsymbol{Q}_{\alpha}\right|$, the pairing is modulated in space as

$$
\Delta(\boldsymbol{r})=\sum_{\alpha}\left|\Delta_{\boldsymbol{Q}_{\alpha}}\right| e^{i \tilde{\theta}_{\alpha}} \cos \left(\frac{1}{2}\left(\theta_{\boldsymbol{Q}_{\alpha}}-\theta_{-\boldsymbol{Q}_{\alpha}}\right)+\boldsymbol{Q}_{\alpha} \cdot \boldsymbol{r}\right)
$$




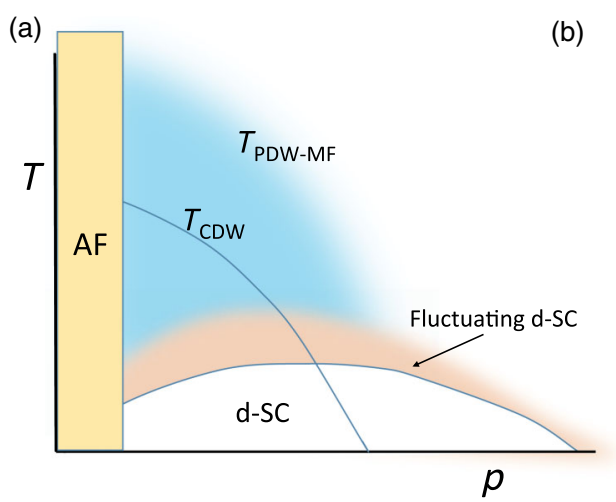

b)

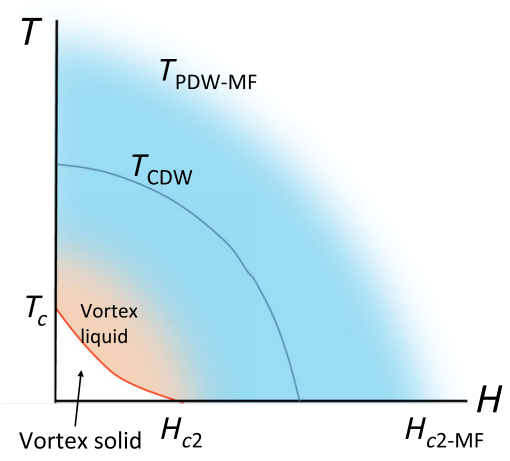

FIG. 7. Proposed phase diagram. (a) $T$ vs doping concentration $p . T_{\text {PDW-MF }}$ is a crossover temperature where the pairing amplitude is established. The phase diagram is dominated by the shaded blue region representing PDW superconductivity, which is short-range ordered because of strong phase fluctuation. It is responsible for the pseudogap. CDW order appears below $T_{\mathrm{CDW}}$. In real material, disorder pinning destroys true long-range order. $d$-wave superconductivity appears below $T_{c}$, accompanied by fluctuating $d$-wave superconductivity which is tied to $T_{c}$. The PDW, either fluctuating or with long-range order, coexists with $d$-wave pairing up to some doping. The precise location and nature of the antiferromagnetic (AF) boundary depends on disorder. (b) $T$ vs magnetic field $H$. The red line is the vortex melting temperature that separates the vortex solid from the vortex liquid. At zero temperature for $H>H_{c 2}$, vortex cores overlap, resulting in a short-range order PDW state that is metallic and shows quantum oscillations. The CDW order is destroyed beyond a certain magnetic field.

where $\tilde{\theta}_{\alpha}=\frac{1}{2}\left(\theta_{Q_{\alpha}}+\theta_{-Q_{\alpha}}\right)$ plays the role of the overall pair phase while $\theta_{\alpha}^{\prime}=\frac{1}{2}\left(\theta_{Q_{\alpha}}-\theta_{-Q_{\alpha}}\right)$ determines the location of the crests of the PDW. The term in the free energy [37]

$$
\begin{aligned}
F= & a\left\{\rho_{2 \boldsymbol{Q}_{1}} \Delta_{\boldsymbol{Q}_{1}}^{*} \Delta_{-\boldsymbol{Q}_{1}}+\rho_{2 \boldsymbol{Q}_{2}} \Delta_{\boldsymbol{Q}_{2}}^{*} \Delta_{-\boldsymbol{Q}_{2}}+\text { c.c. }\right\} \\
& +a^{\prime}\left\{\rho_{\boldsymbol{Q}_{1}+\boldsymbol{Q}_{2}}\left(\Delta_{\boldsymbol{Q}_{1}}^{*} \Delta_{-\boldsymbol{Q}_{2}}+\Delta_{\boldsymbol{Q}_{2}}^{*} \Delta_{-\boldsymbol{Q}_{1}}\right)+\text { c.c. }\right\}
\end{aligned}
$$

is the phenomenological basis for generating the induced order $\rho_{2 \boldsymbol{Q}_{1}}, \rho_{2 \boldsymbol{Q}_{2}}$, and $\rho_{\boldsymbol{Q}_{1}+\boldsymbol{Q}_{2}}[19,44,25]$. It turns out that unlike $\rho_{2 \boldsymbol{Q}_{1}}$ and $\rho_{2 \boldsymbol{Q}_{2}}$, the CDW $\rho_{\boldsymbol{Q}_{1}+\boldsymbol{Q}_{2}}$ is not necessarily generated [37]. We defer the discussion of this to later. Equation (8) generates locking between the phases of the form $\cos \left(\theta_{Q_{\alpha}}-\theta_{-Q_{\alpha}}-\phi_{\alpha}\right)$. $\tilde{\theta}_{\alpha}$ and $\theta_{\alpha}^{\prime}$ appear to be independent, but there are subtleties associated with vortex formation $[25,44]$. Nevertheless, it is possible that phase fluctuations produced by conventional $h c / 2 e$ vortices can destroy the $\tilde{\theta}_{\alpha}$ correlation while leaving $\theta_{\alpha}^{\prime}$ intact. Thus, it is possible that the induced CDW order has long-range order while the pairing is short range [45]. In reality, disorder pinning limits the spatial order. For incommensurate $Q, \theta_{\alpha}^{\prime}$ can be set to zero by choosing the origin of $\boldsymbol{r}$, and the locking term becomes simply $\cos \phi_{\alpha}$. Physically, $\phi_{\alpha}$ describes the relative location of the crests of the PDW and CDW. When it is locked to 0 or $\pi$, reflection symmetry is preserved in the plane. A deviation of $\phi_{\alpha}$ from 0 or $\pi$ can come from higher-order terms in the free energy of the form $b\left(\rho_{2 \boldsymbol{Q}_{\alpha}} \Delta_{\boldsymbol{Q}_{\alpha}} \Delta_{-\boldsymbol{Q}_{\alpha}}^{*}\right)^{2}+$ c.c. etc., which gives a locking term $b \cos 2 \phi_{\alpha}$. If $a$ and $b$ are opposite in sign and $|b|>|a| / 4$, the free energy has a minimum at $\phi_{\alpha}= \pm \phi_{0}$, thereby breaking the reflection symmetry in the plane. To break reflection symmetry along $z$, note that the stacking in the $z$ direction can take on two distinct forms: $\left(\phi_{1}, \phi_{2}\right)=$ $\left[\left(\phi_{0}, \phi_{0}\right),\left(\phi_{0},-\phi_{0}\right),\left(-\phi_{0},-\phi_{0}\right),\left(-\phi_{0}, \phi_{0}\right)\right]$ or $\left[\left(\phi_{0}, \phi_{0}\right)\right.$, $\left.\left(-\phi_{0}, \phi_{0}\right),\left(-\phi_{0},-\phi_{0}\right),\left(\phi_{0},-\phi_{0}\right)\right]$, thereby introducing a sense of chirality. While we do not have a microscopic basis for the appearance of a finite $\phi_{0}$, this argument at least offers the possibility that a gyrotropic state is associated with Amperean pairing and checkerboard CDW order.

Is there a way to directly detect the PDW? The pairing response function $\chi(q, \omega)$ can be measured [46] by constructing a tunnel junction between an optimally doped cuprate superconductor and a material with a low $T_{c}$, such as $\mathrm{Bi}-2201$, and can operate at a temperature between the two $T_{c}$ 's. The pair momentum is supplied by a parallel magnetic field. The tunneling current vs magnetic field and voltage is predicted to be proportional to $\operatorname{Im} \chi(q, \omega)$, where $\omega=2 e V / \hbar$ and $q=(2 e B / \hbar c)(\lambda+d / 2)$, with $\lambda$ the penetration depth of the superconductor (assumed to be thick) and $d$ the barrier thickness. The fluctuating PDW will give rise to a peak in the tunneling current at $B$ corresponding to $q= \pm Q$. The same experiment can be performed at low temperatures by using a $d$-wave or conventional $s$-wave superconductor. If the PDW coexists with $d$-wave pairing, a peak in the current is predicted to emerge at a field far greater than that expected for the Fraunhofer pattern coming from the $d$-wave order. The shape of this peak will contain information about the degree of the PDW order. This kind of tunneling structure has in fact been fabricated and studied in the absence of a magnetic field [47]. Hence, the proposed experiment should be quite feasible.

Finally, we discuss other orders that may appear in this scenario. The Landau theory without phase fluctuation with the symmetry of a square lattice has been discussed by Agterberg and Tsunetsugu [37], and we quote their results. 
In the checkerboard case, there is a term in the Landau free energy that locks the phase $\left(\theta_{\boldsymbol{Q}_{1}}+\theta_{-\boldsymbol{Q}_{1}}\right)-\left(\theta_{\boldsymbol{Q}_{2}}+\theta_{-\boldsymbol{Q}_{2}}\right)$ to be 0 or $\pi$. The resulting states are called case 4 and 5 , respectively, in Ref. [37]. In case 4, the last term in Eq. (8) is allowed and $\rho_{\boldsymbol{Q}_{1} \pm \boldsymbol{Q}_{2}}$ is generated. However, in case 5 , this term cancels and CDW at $\boldsymbol{Q}_{1} \pm \boldsymbol{Q}_{2}$ is not expected. Instead, a magnetization wave at $\boldsymbol{Q}_{1} \pm \boldsymbol{Q}_{2}$ is predicted. The $\boldsymbol{Q}_{1} \pm$ $\boldsymbol{Q}_{2}$ CDW peak has been searched for but so far not found [48]. This suggests that we may be in case 5, and it will be interesting to search for orbital magnetism at wave vector $\boldsymbol{Q}_{1} \pm \boldsymbol{Q}_{2}$.

We now discuss the coexistence of PDW with $d$-wave order $\Delta_{d}$. We find that the PDW may develop long-range order (limited by disorder) even when phase fluctuations are included. The reason for this is that a term $\left(\Delta_{d}^{*}\right)^{2}\left(\Delta_{Q_{1}} \Delta_{-Q_{1}}+\Delta_{Q_{2}} \Delta_{-} Q_{2}\right)+$ c.c. is allowed. This term pins the phase $\theta_{\boldsymbol{Q}_{\alpha}}+\theta_{-\boldsymbol{Q}_{\alpha}}$ (the pinning is much weaker in case 5 than in case 4), and since $\theta_{\boldsymbol{Q}_{\alpha}}-\theta_{-\boldsymbol{Q}_{\alpha}}$ is assumed to be locked already, the individual phases $\theta_{\boldsymbol{Q}_{\alpha}}$ will be pinned up to $\pi$. Thus, $\Delta_{Q_{\alpha}}$ may have long-range order, in the phase where domain walls with $\pi$ phase shifts are not important. In this case, the term in the Landau free energy $\rho_{\boldsymbol{Q}_{\alpha}} \Delta_{\boldsymbol{Q}_{\alpha}} \Delta_{d}^{*}$ implies that CDWs with wave vectors $\boldsymbol{Q}_{\alpha}$ are predicted to appear in the superconducting state. It is intriguing that STM measurements at $6 \mathrm{~K}$ on optimally doped YBCO have found charge ordering with two sets of ordering vectors at $\delta=0.28 \pm 0.03$ and $0.14 \pm 0.01$ (in units of $2 \pi$ ) along the $x$ and $y$ axes [49]. It is tempting to reinterpret these CDWs to correspond to our $\rho_{2 \boldsymbol{Q}_{\alpha}}$ and $\rho_{\boldsymbol{Q}_{\alpha}}$, respectively. Clearly, more work needs to be done to confirm this interpretation, particularly since density waves at these half wave vectors have not been reported by x-ray scattering.

In conclusion, recent experimental advances have put severe constraints on the nature of the pseudogap state. The assumption of Amperean pairing gives a consistent account of all the unusual phenomena. It remains to be fully understood why long-range order is not achieved below $T^{*}$ and above a small " $H_{c 2}$." Phase fluctuations are presumably at play, but a detailed thermal and quantum description of the short-range-ordered state will be highly desirable. If it is possible to increase the interlayer Josephson tunneling by building artificial MBE structures, we expect that phase fluctuations will be suppressed, and it will be extremely interesting to see if a long-range-ordered Amperean superconducting state at a relatively high temperature between $T_{c}$ and $T^{*}$ can be stabilized.

\section{ACKNOWLEDGMENTS}

I thank N. Phuan Ong for emphasizing to me the pairing nature of the pseudogap phase and for sharing his insight that the high-field state is some form of PDW. I also thank T. Senthil for many discussions on the high- $T_{c}$ topic. I acknowledge support from NSF Grant No. DMR-1104498.

\section{APPENDIX A: INCOMPATIBILITY OF THE CDW MODEL WITH THE ARPES DATA}

With the discovery of CDW order, it seems natural to associate the energy gap induced by CDW with the pseudogap [50-53]. Here, we show that a mean-field picture of the CDW fails to explain the ARPES data of He et al. [12]. Figure 8 shows several scans of the spectrum along $k_{x}$ starting from the antinode, as indicated in Fig. 1. By a judicial choice of the CDW ordering vector $\delta$ and the gap size, the scan at the antinode can account for the ARPES data reproduced in Fig. 3(b). However, the agreement breaks down away from the antinode. Figures 8(b) and 8 (c) show the spectrum for $\delta k_{y}$ near the appearance of the Fermi arc and in the middle of the Fermi arc where the band crosses the Fermi level. It is clear that the Fermi arc is formed by a state moving down towards the Fermi level, leaving a large gap just below. This is in strong contradiction with the data shown in Fig. 3(d), which shows that the gap is closed by a state moving up in energy to meet the Fermi level. In particular, the CDW model predicts a large gap below the Fermi level at the end of the Fermi arc, which has never been seen experimentally. In fact, it has been emphasized that a gap exists above the Fermi level near the end of the Fermi arc [36].
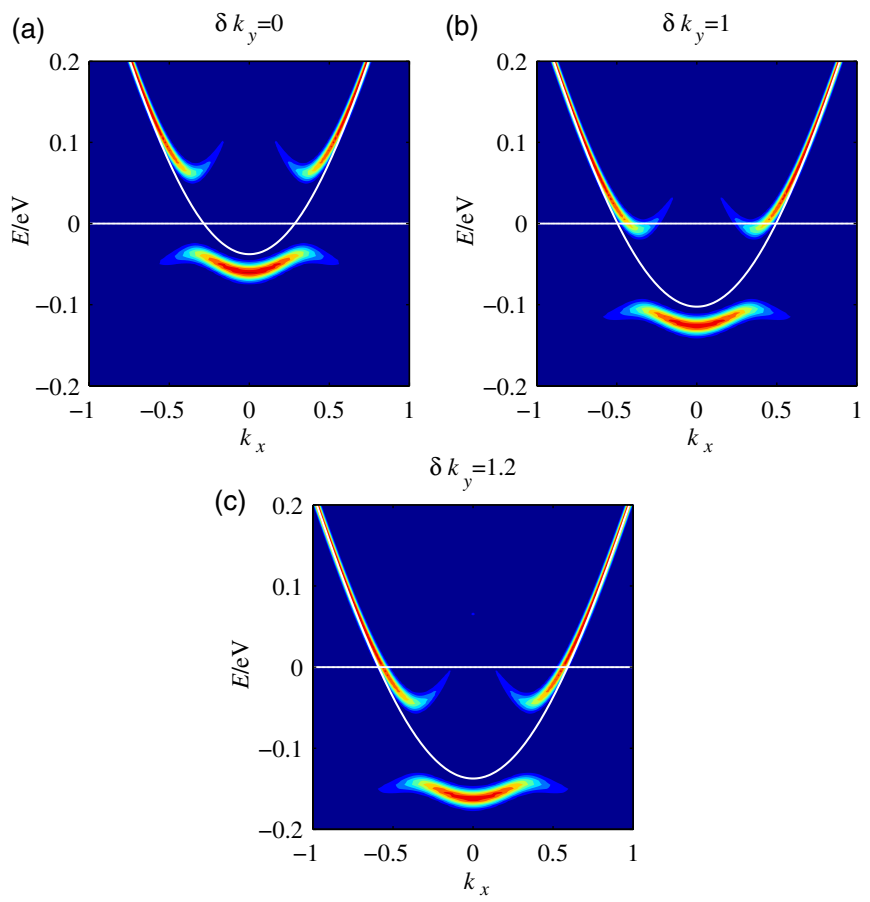

FIG. 8. Plot of the energy spectrum vs $k_{x}$ for (a) $k_{y}=\pi$, $\delta k_{y}=0$, (b) $\delta k_{y}=1.0$, and (c) $\delta k_{y}=1.2$. The CDW wave vector is assumed to be $1.2 Q$, and the gap is set to be $0.05 \mathrm{eV}$. Panel (c) corresponds to a cut through the Fermi arc, while panel (b) is a cut near the end of the arc. Note that the state at the Fermi level arises by a state moving from above, leaving a gap below the Fermi level, in strong disagreement with the data shown in Figs. 3(b)-3(e). 
We note that Ref. [53] assumes an interference between the CDWs in the $x$ and $y$ directions, so the gap vanishes at the "hot spot." However, the gap reopens away from the hot spot and should be visible below the Fermi level in the Fermi arc region. Alternatively, in the bond density wave picture, the gap is $\boldsymbol{k}$ dependent and vanishes at the node [50-52]. However, the difficulty described here remains as long as the gap is nonzero at the end of the Fermi arc.

\section{APPENDIX B: DETAILS OF THE ENERGY SPECTRA}

We show in Fig. 9 the dispersion and the spectral weight of the solution of the five-band model defined by Eq. (5) for $k_{y}=\pi$. Note the common tangents of the original bare band with the hole bands (solid and dashed lines), which give rise to large gaps at the Fermi level. At $k_{x}=0$, the bare hole bands cross at $-100 \mathrm{meV}$. One linear combination is

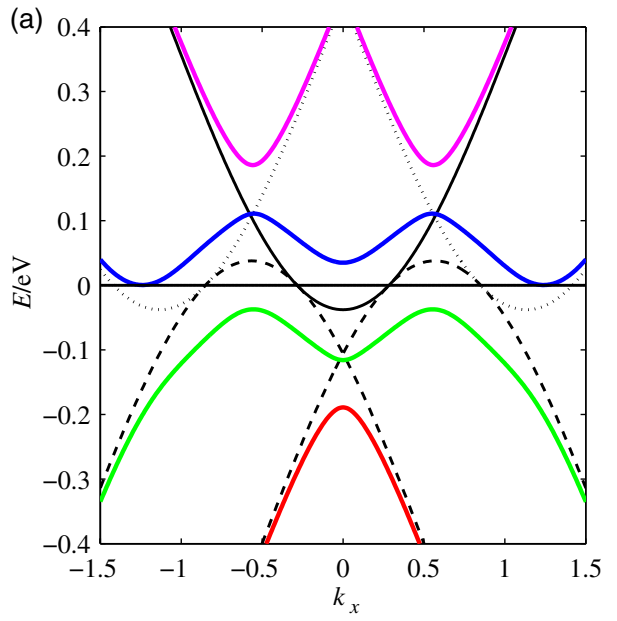

decoupled from the electron band, and the dispersion of the green band goes through this point, albeit with zero spectral weight because it is decoupled from the electron. This feature can also be seen in the three-band spectrum shown in Fig. 2. Figures 10-12 show how the band structure evolves as $k_{y}$ deviates from $\pi$. The top of the green band moves towards the Fermi level and crosses it near $\delta k_{y}=0.6$. Beyond that, it forms what looks like a pocket [see Fig. 11(a)], but the back side of the pocket is mainly a holelike quasiparticle, so its spectral weight for removing an electron is so small that it is not visible in Fig. 4. Instead, what is seen is a bending of the "Fermi surface" away from the bare Fermi surface (see Fig. 4). We also note that because of the repulsion by the electron bands shifted by $\pm 2 Q$ (dotted lines), which crosses the original bare band at $k_{x}= \pm Q$, the top of the green band is located near $k_{x}= \pm Q$. As a result, the tip of the Fermi arc, as defined by the green band touching the Fermi level, lies close to $k_{x}= \pm Q$. Consequently, the

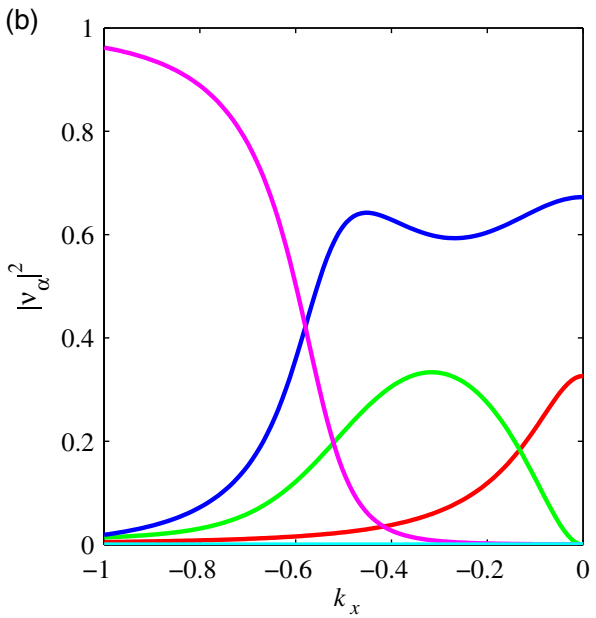

FIG. 9. (a) Plot of the energy bands of the five-band model defined by Eq. (5) for $k_{y}=\pi$. The dashed lines show $-\left(\varepsilon_{-k \pm \mathbf{Q}}-\mu\right)$. The dotted line shows $\left(\varepsilon_{k \pm 2 Q}-\mu\right)$. (b) The spectral weight of the bands. The parameters used are the same as in Fig. 3, with the band parameters taken from Ref. [12].
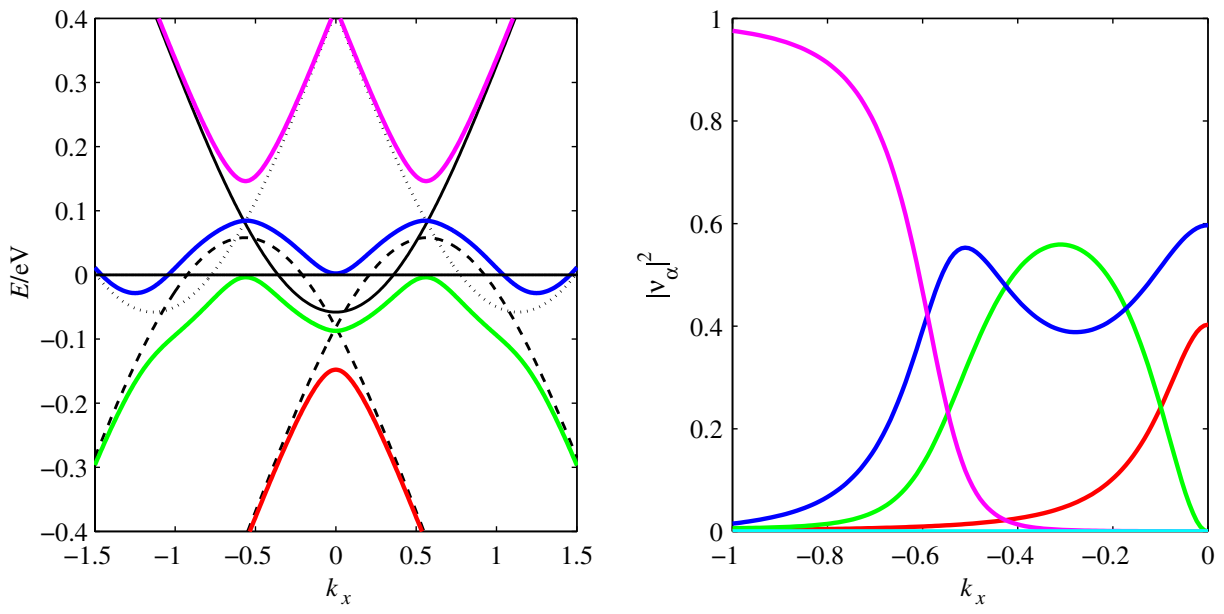

FIG. 10. Same as Fig. 9 except that $\delta k_{y}=0.6$. 

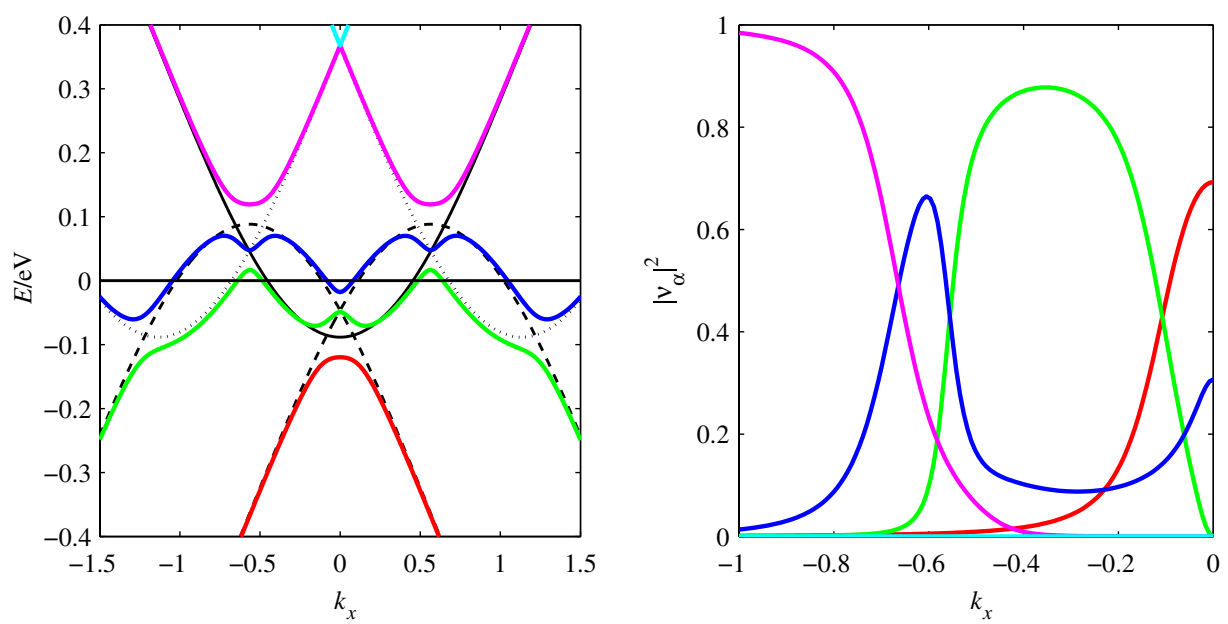

FIG. 11. Same as Fig. 9 except that $\delta k_{y}=0.9$.
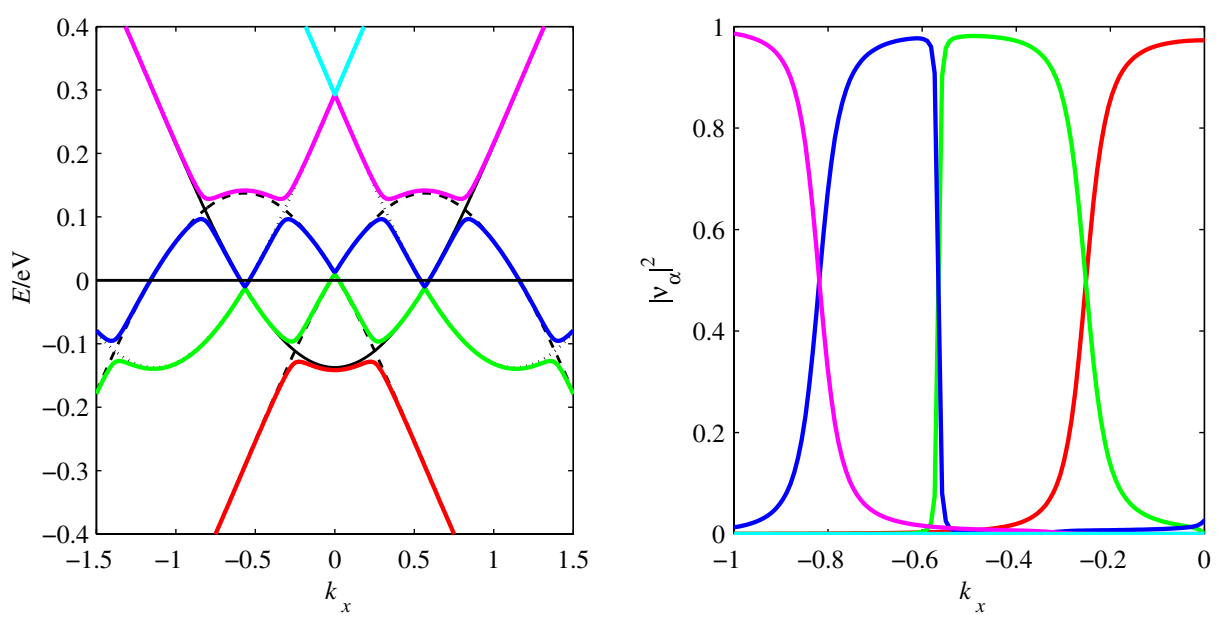

FIG. 12. Same as Fig. 9 except that $\delta k_{y}=1.2$.

spanning vector of the tips of the arcs is $2 \mathrm{Q}$, which is also the CDW wave vector $\delta$ in our theory.

Note that at the crossing at $k_{x}= \pm Q$ mentioned above between the original band and one shifted by $2 Q$ (black and dotted lines in the figures), one combination is decoupled, thereby pinning the blue band to the crossing point. As $k_{y}$ continues to move away from $\pi$, the crossing point comes down in energy and collides with the top of the green band. (a)

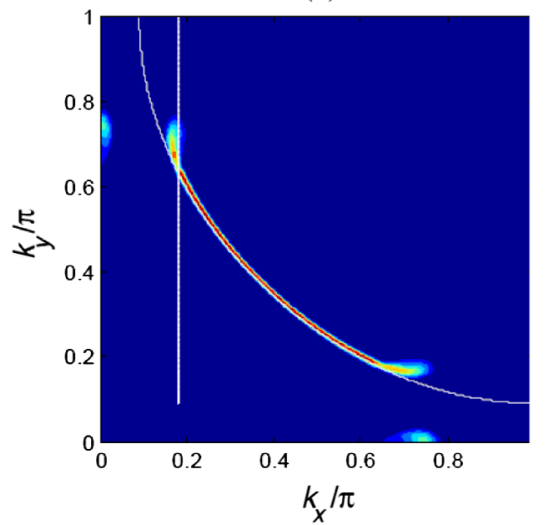

(b)

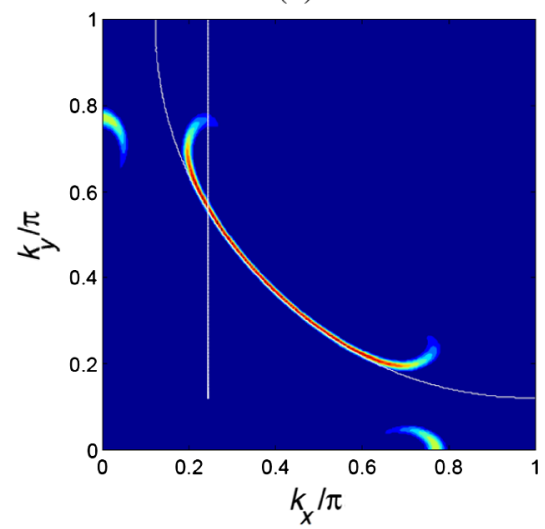

(c)

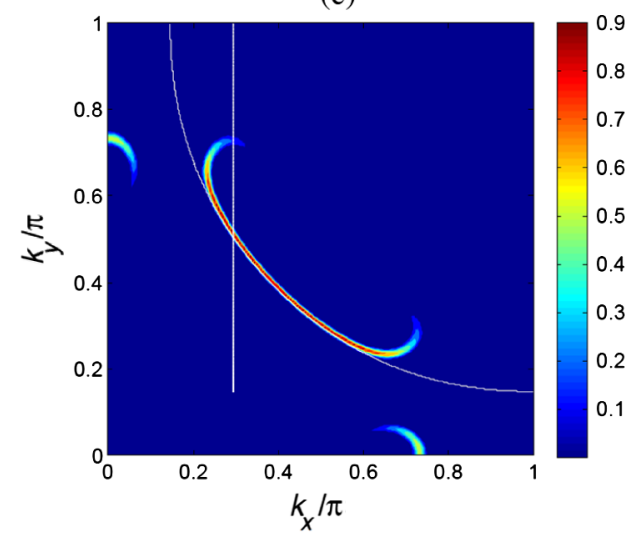

FIG. 13. Spectral weight at the Fermi level. Same as Fig. 4 except that (a) $\Delta_{0}=100 \mathrm{meV}, p=0.207$, (b) $p=0.157$ and $\Delta_{0}=150 \mathrm{meV}$, (c) $p=0.112$ and $\Delta_{0}=250 \mathrm{meV}$. 
This happens near $\delta k_{y}=1.2$ [Fig. 12(a)] and produces a very small gap. Judging from the spectral weight shown in Fig. 12(b), the bands are essentially crossing without much coupling, and a robust Fermi surface arc is in place beyond this point. The position where the bare band crossing crosses the Fermi level is the point where the vertical white line crosses the bare Fermi surface shown in Fig. 4. We emphasize that so far we have set the direct coupling to the CDW to zero so that the gap at the tip of the arc is a weak induced gap through the pairing. In Fig. 13, we show the spectral weight of the Fermi surface for different doping, demonstrating how the Fermi arc shrinks with underdoping.

The energy spectrum for a PDW was calculated by Baruch and Orgad [33]. They considered a unidirectional PDW with a wave vector $\delta$ which is not tied to the Fermi surface spanning vector $Q$ but is close to it. For a sufficiently large gap, the qualitative feature near $(0, \pi)$ is similar to ours. However, they assume a pairing amplitude that is independent of $k_{y}$, whereas we assume that it is large only near $(0, \pi)$. As a result, they also find a gap structure near $(\pi, 0)$, while we do not if we treat a unidirectional CDW.

[1] For a review of experimental data, see P. A. Lee, N. Nagaosa, and X.-G. Wen, Doping a Mott Insulator: Physics of High-Temperature Superconductivity, Rev. Mod. Phys. 78, 17 (2006).

[2] G. Ghiringhelli, M. Le Tacon, M. Minola, S. BlancoCanosa, C. Mazzoli, N. B. Brookes, G. M. De Luca, A. Frano, D. G. Hawthorn, F. He, T. Loew, M. Moretti Sala, D. C. Peets, M. Salluzzo, E. Schierle, R. Sutarto, G. A. Sawatzky, E. Weschke, B. Keimer, and L. Braicovich, Long-Range Incommensurate Charge Fluctuations in (Y, Nd) $\mathrm{Ba}_{2} \mathrm{Cu}_{3} \mathrm{O}_{6+x}$, Science 337, 821 (2012).

[3] J. Chang, E. Blackburn, A. T. Holmes, N. B. Christensen, J. Larsen, J. Mesot, Ruixing Liang, D. A. Bonn, W. N. Hardy, A. Watenphul, M. v. Zimmermann, E. M. Forgan, and S. M. Hayden, Direct Observation of Competition Between Superconductivity and Charge Density Wave Order in $\mathrm{YBa}_{2} \mathrm{Cu}_{3} \mathrm{O}_{6.67}$, Nat. Phys. 8, 871 (2012).

[4] M. Le Tacon, A. Bosak, S. M. Souliou, G. Dellea, T. Loew, R. Heid, K-P. Bohnen, G. Ghiringhelli, M. Krisch, and B. Keimer, Inelastic X-ray Scattering in $\mathrm{YBa}_{2} \mathrm{Cu}_{3} \mathrm{O}_{6.6}$ Reveals Giant Phonon Anomalies and Elastic Central Peak Due to Charge-Density-Wave Vormation, Nat. Phys. 10, 52 (2014).

[5] R. Comin, A. Frano, M. M. Yee, Y. Yoshida, H. Eisaki, E. Schierle, E. Weschke, R. Sutarto, F. He, A. Soumyanarayanan, Y. He, M. Le Tacon, I. S. Elfimov, J. E. Hoffman, G. A. Sawatzky, B. Keimer, and A. Damascelli, Charge Order Driven by Fermi-Arc Instability in $\mathrm{Bi}_{2} \mathrm{Sr}_{2-\mathrm{x}} \mathrm{La}_{\mathrm{x}} \mathrm{CuO}_{6+\delta}$, Science 343, 390 (2014).

[6] C. Howald, H. Eisaki, N. Kaneko, and A. Kapitulnik, Coexistence of Periodic Modulation of Quasiparticle States and Superconductivity in $\mathrm{Bi}_{2} \mathrm{Sr}_{2} \mathrm{CaCu}_{2} \mathrm{O}_{8+\delta}$, Proc. Natl. Acad. Sci. U.S.A. 100, 9705 (2003).

[7] M. Vershinin, S. Misra, S. Ono, Y. Abe, Y. Ando, and A. Yazdani, Local Ordering in the Pseudogap State of the High- $T_{c}$ Superconductor $\mathrm{Bi}_{2} \mathrm{Sr}_{2} \mathrm{CaCu}_{2} \mathrm{O}_{8+\delta}$, Science 303, 1995 (2004).

[8] T. Hanaguri, C. Lupien, Y. Kohsaka, D. H. Lee, M. Azuma, M. Takano, H. Takagi, and J. C. Davis, A "Checkerboard" Electronic Crystal State in Lightly Hole-Doped $\mathrm{Ca}_{2-\mathrm{x}} \mathrm{Na}_{\mathrm{x}} \mathrm{CuO}_{2} \mathrm{Cl}_{2}$, Nature (London) 430, 1001 (2004).

[9] W. D. Wise, M. C. Boyer, K. Chatterjee, T. Kondo, T. Takeuchi, H. Ikuta, Y. Wang, and E. W. Hudson, ChargeDensity-Wave Origin of Cuprate Checkerboard Visualized by Scanning Tunnelling Microscopy, Nat. Phys. 4, 696 (2008).

[10] T. Wu, H. Mayaffre, S. Kramer, M. Horvatic, C. Berthier, W. N. Hardy, R. Liang, D. A. Bonn, and M.-H. Julien, Magnetic-Field-Induced Charge-Stripe Order in the High-Temperature Superconductor $\mathrm{YBa}_{2} \mathrm{Cu}_{3} \mathrm{O}_{\mathrm{y}}$, Nature (London) 477, 191 (2011).

[11] E. H. da Silva Neto, P. Aynajian, A. Frano, R. Comin, E. Schierle, E. Weschke, A. Gyenis, J. Wen, J. Schneeloch, Z. $\mathrm{Xu}$, S. Ono, G. Gu, M. Le Tacon, and A. Yazdani, Ubiquitous Interplay Between Charge Ordering and High-Temperature Superconductivity in Cuprates, Science 343, 393 (2014).

[12] R.-H. He, M. Hashimoto, H. Karapetyan, J. D. Koralek, J. P. Hinton, J. P. Testaud, V. Nathan, Y. Yoshida, H. Yao, K. Tanaka, W. Meevasana, R. G. Moore, D. H. Lu, S.-K. Mo, M. Ishikado, H. Eisaki, Z. Hussain, T. P. Devereaux, S. A. Kivelson, J. Orenstein, A. Kapitulnik, and Z.-X. Shen, From a Single-Band Metal to a High-Temperature Superconductor via Two Thermal Phase Transitions, Science 331, 1579 (2011).

[13] R. Daou, J. Chang, D. LeBoeuf, O. Cyr-Choiniere, F. Laliberte, N. Doiron-Leyraud, B. J. Ramshaw, R. Liang, D. A. Bonn, W. N. Hardy, and L. Taillefer, Broken Rotational Symmetry in the Pseudogap Phase of a High- $T_{c}$ Superconductor, Nature (London) 463, 519 (2010).

[14] P. Bourges and Y. Sidis, Novel Magnetic Order in the Pseudogap State of High- $\mathrm{T}_{c}$ Copper Oxides Superconductors, C.R. Phys. 12, 461 (2011).

[15] N. Doiron-Leyraud, C. Proust, D. LeBoeuf, J. Levallois, J.-B. Bonnemaison, Ruixing Liang, D. A. Bonn, W. N. Hardy, and L. Taillefer, Quantum Oscillations and the Fermi Surface in an Underdoped High- $T_{c}$ Superconductor, Nature (London) 447, 565 (2007).

[16] G. Grissonnanche, O. Cyr-Choiniere, F. Laliberte, S. Rene de Cotret, A. Juneau-Fecteau, S. Dufour-Beausejour, M. E. Delage, D. LeBoeuf, J. Chang, B. J. Ramshaw, D. A. Bonn, W. N. Hardy, R. Liang, S. Adachi, N. E. Hussey, B. Vignolle, C. Proust, M. Sutherland, S. Kramer, J. H. Park, D. Graf, N. Doiron-Leyraud, and L. Taillefer, Direct Measurement of the Upper Critical Field in Cuprate Superconductors, Nat. Commun. 5, 3280 (2014).

[17] L. Li, Y. Wang, S. Komiya, S. Ono, Y. Ando, G. D. Gu, and N. P. Ong, Diamagnetism and Cooper Pairing above $T_{c}$ in cuprates, Phys. Rev. B 81, 054510 (2010).

[18] A. Dubroka, M. Rössle, K. W. Kim, V. K. Malik, D. Munzar, D. N. Basov, A. A. Schafgans, S. J. Moon, 
C. T. Lin, D. Haug, V. Hinkov, B. Keimer, Th. Wolf, J. G. Storey, J. L. Tallon, and C. Bernhard, Evidence of a Precursor Superconducting Phase at Temperatures as High as $180 \mathrm{~K}$ in $\mathrm{RBa}_{2} \mathrm{Cu}_{3} \mathrm{O}_{7-\delta}(\mathrm{R}=\mathrm{Y}, \mathrm{GD}, \mathrm{Eu})$ Superconducting Crystals from Infrared Spectroscopy, Phys. Rev. Lett. 106, 047006 (2011).

[19] S.-S. Lee, P. A. Lee, and T. Senthil, Amperean Pairing Instability in the U(1) Spin Liquid State with Fermi Surface and Application to $\kappa-(\mathrm{BEDT}-\mathrm{TTF})_{2} \mathrm{Cu}_{2}(\mathrm{CN})_{3}$, Phys. Rev. Lett. 98, 067006 (2007).

[20] T. Senthil and P. A. Lee, Coherence and Pairing in a Doped Mott Insulator: Application to the Cuprates, Phys. Rev. Lett. 103, 076402 (2009).

[21] G. Kotliar and J. Liu, Superexchange Mechanism and $d$-wave Superconductivity, Phys. Rev. B 38, 5142 (1988).

[22] P. Corboz, T. M. Rice, and M. Troyer, Competing States in the t-J model: Uniform d-Wave State Versus Stripe State, arXiv:1402.2859 [Phys. Rev. Lett. (to be published)].

[23] Strictly speaking, the assumption that the PDW momentum is $2 \mathrm{~K}$ is justified for weak coupling. For strong coupling, we may expect a small deviation.

[24] H.-D. Chen, O. Vafek, A. Yazdani, and S.-C. Zhang, Pair Density Wave in the Pseudogap State of High Temperature Superconductors, Phys. Rev. Lett. 93, 187002 (2004). This version of PDW has a period that is the same as the CDW and is different from other examples discussed here.

[25] E. Berg, E. Fradkin, S. A. Kivelson, and J. M. Tranquada, Striped Superconductors: How Spin, Charge and Superconducting Orders Intertwine in the Cuprates, New J. Phys. 11, 115004 (2009), and references therein.

[26] P. Fulde and R. A. Ferrell, Superconductivity in a Strong Spin-Exchange Field, Phys. Rev. 135, A550 (1964).

[27] A. I. Larkin, and Yu. N. Ovchinnikov, Inhomogeneous State of Superconductors, Sov. Phys. JETP 20, 762 (1965).

[28] Q. Li, M. Hücker, G. D. Gu, A. M. Tsvelik, and J. M. Tranquada, Two-Dimensional Superconducting Fluctuations in Stripe-Ordered $\mathrm{La}_{1.875} \mathrm{Ba}_{0.125} \mathrm{CuO}_{4}$, Phys. Rev. Lett. 99, 067001 (2007).

[29] E. Berg, E. Fradkin, E.-A. Kim, S. A. Kivelson, V. Oganesyan, J. M. Tranquada, and S. C. Zhang, Dynamical Layer Decoupling in a Stripe-Ordered High- $T_{c}$ Superconductor, Phys. Rev. Lett. 99, 127003 (2007).

[30] A. Himeda, T. Kato, and M. Ogata, Stripe States with Spatially Oscillating $d$-wave Superconductivity in the Two-Dimensional $\mathrm{t}-\mathrm{t}^{\prime}-\mathrm{J}$ Model, Phys. Rev. Lett. 88, 117001 (2002).

[31] M. Zelli, C. Kallin, and A. John Berlinsky, Quantum Oscillations in a $\pi$-Striped Superconductor, Phys. Rev. B 86, 104507 (2012).

[32] F. Yu, M. Hirschberger, T. Loew, G. Li, B. J. Lawson, T. Asaba, J. B. Kemper, T. Liang, J. Porras, G. S. Boebinger, J. Singleton, B. Keimer, L. Li, and N. P. Ong, Diamagnetic Response in Under-Doped $\mathrm{YBa}_{2} \mathrm{Cu}_{3} \mathrm{O}_{6.6}$ in High Magnetic Fields, arXiv:1402.7371.

[33] The spectra for PDW states have been studied by S. Baruch and D. Orgad, Spectral Signatures of Modulated d-Wave Superconducting Phases, Phys. Rev. B 77, 174502 (2008) and in Ref. [25]. Similarities to and differences from the present work are discussed in Appendix B.
[34] The absence of an energy gap is consistent with our argument that the spin susceptibility is not changed by Amperean pairing [C. P. Nave, S.-S. Lee, and P. A. Lee, Susceptibility of a Spinon Fermi Surface Coupled to a U(1) Gauge Field, Phys. Rev. B 76, 165104 (2007)]. However, this argument is valid only in the limit of a small order parameter. Once a gap is formed with a sufficiently large $\Delta_{Q}$, we expect that the spin susceptibility will be reduced.

[35] Y. Kohsaka, C. Taylor, P. Wahl, A. Schmidt, Jhinhwan Lee, K. Fujita, J. W. Alldredge, K. McElroy, Jinho Lee, H. Eisaki, S. Uchida, D. H. Lee, and J. C. Davis, How Cooper Pairs Vanish Approaching the Mott Insulator in $\mathrm{Bi}_{2} \mathrm{Sr}_{2} \mathrm{CaCu}_{2} \mathrm{O}_{8+\delta}$, Nature (London) 454, 1072 (2008).

[36] H.-B. Yang, J. D. Rameau, Z.-H. Pan, G. D. Gu, P. D. Johnson, H. Claus, D. G. Hinks, and T. E. Kidd, Reconstructed Fermi Surface of Underdoped $\mathrm{Bi}_{2} \mathrm{Sr}_{2} \mathrm{CaCu}_{2} \mathrm{O}_{8+\delta}$ Cuprate Superconductors, Phys. Rev. Lett. 107, 047003 (2011).

[37] D. F. Agterberg and H. Tsunetsugu, Dislocations and Vortices in Pair-Density-Wave Superconductors, Nat. Phys. 4, 639 (2008).

[38] E. Blackburn, J. Chang, M. Hücker, A. T. Holmes, N. B. Christensen, Ruixing Liang, D. A. Bonn, W. N. Hardy, U. Rütt, O. Gutowski, M. v. Zimmermann, E. M. Forgan, and S. M. Hayden, X-Ray Diffraction Observations of a ChargeDensity-Wave Order in Superconducting Ortho-II $\mathrm{YBa}_{2} \mathrm{Cu}_{3} \mathrm{O}_{6.54}$ Single Crystals in Zero Magnetic Field, Phys. Rev. Lett. 110, 137004 (2013).

[39] S. Blanco-Canosa, A. Frano, T. Loew, Y. Lu, J. Porras, G. Ghiringhelli, M. Minola, C. Mazzoli, L. Braicovich, E. Schierle, E. Weschke, M. Le Tacon, and B. Keimer, Momentum-Dependent Charge Correlations in $\mathrm{YBa}_{2} \mathrm{Cu}_{3} \mathrm{O}_{6+\delta}$ Superconductors Probed by Resonant $X$ Ray Scattering: Evidence for Three Competing Phases, Phys. Rev. Lett. 110, 187001 (2013).

[40] J. E. Hoffman, E. W. Hudson, K. M. Lang, V. Madhavan, H. Eisaki, S. Uchida, and J.C. Davis, A Four Unit Cell Periodic Pattern of Quasi-Particle States Surrounding Vortex Cores in $\mathrm{Bi}_{2} \mathrm{Sr}_{2} \mathrm{CaCu}_{2} \mathrm{O}_{8+\delta}$, Science 295, 466 (2002).

[41] N. Harrison and S. E. Sebastian, Protected Nodal Electron Pocket from Multiple-Q Ordering in Underdoped High Temperature Superconductors, Phys. Rev. Lett. 106, 226402 (2011).

[42] N. Barisic, S. Badoux, M. K. Chan, C. Dorow, W. Tabis, B. Vignolle, G. Yu, J. Beard, X. Zhao, C. Proust, and M. Greven, Universal Quantum Oscillations in the Underdoped Cuprate Superconductors, Nat. Phys. 9, 761 (2013).

[43] P. Hosur, A. Kapitulnik, S. A. Kivelson, J. Orenstein, and S. Raghu, Kerr Effect as Evidence of Gyrotropic Order in the Cuprates, Phys. Rev. B 87, 115116 (2013).

[44] P. Hosur, A. Kapitulnik, S. A. Kivelson, J. Orenstein, S. Raghu, W. Cho, and A. Fried, Kerr Effect as Evidence of Gyrotropic Order in the Cuprates-Revisited, arXiv:1405.0752.

[45] E. Berg, E. Fradkin, and S. A. Kivelson, Charge-4e Superconductivity from Pair-Density-Wave Order in Certain High-Temperature Superconductors, Nat. Phys. 5, 830 (2009).

[46] D. J. Scalapino, Pair Tunneling as a Probe of Fluctuations in Superconductors, Phys. Rev. Lett. 24, 1052 (1970). 
[47] N. Bergeal, J. Lesueur, M. Aprili, G. Faini, J. P. Contour, and B. Leridon, Pairing Fluctuations in the Pseudogap State of Copper-Oxide Superconductors Probed by the Josephson Effect, Nat. Phys. 4, 608 (2008).

[48] R. Comin, R. Sutarto, F. He, E. da Silva Neto, L. Chauviere, A. Frano, R. Liang, W. N. Hardy, D. Bonn, Y. Yoshida, H. Eisaki, J. E. Hoffman, B. Keimer, G. A. Sawatzky, and A. Damascelli, The Symmetry of Charge Order in Cuprates, arXiv: 1402.5415 .

[49] N.-C. Yeh and A. D. Beyer, Unconventional Low-Energy Excitations of Cuprate Superconductors, Int. J. Mod. Phys. B 23, 4543 (2009).
[50] M. A. Metlitski and S. Sachdev, Quantum Phase Transitions of Metals in Two Spatial Dimensions. II. Spin Density Wave Order, Phys. Rev. B 82, 075128 (2010).

[51] S. Sachdev and R. La Placa, Bond Order in TwoDimensional Metals with Antiferromagnetic Exchange Interactions, Phys. Rev. Lett. 111, 027202 (2013).

[52] Y. Wang and A. V. Chubukov, Charge Order and Loop Currents in Hole-Doped Cuprates, arXiv:1401.0712.

[53] J.C. Séamus Davis and D.-H. Lee, Concepts Relating Magnetic Interactions, Intertwined Electronic Orders, and Strongly Correlated Superconductivity, Proc. Nat. Acad. Sci. 110, 17623 (2013). 